\title{
Prefrontal Inositol Triphosphate Is Molecular Correlate of Working Memory in Nonhuman Primates
}

\author{
Juan F. López-Téllez, ${ }^{1,2,5}$ Manuel F. López-Aranda, ${ }^{1,2,5}$ Irene Navarro-Lobato, ${ }^{1,2}$ Mariam Masmudi-Martín,, \\ Elisa Martín Montañez, ${ }^{1,3}$ Eduardo Blanco Calvo, ${ }^{4}$ and Zafar U. Khan ${ }^{1,2,5}$ \\ ${ }^{1}$ Laboratory of Neurobiology at Centro de Investigaciones Médico Sanitarias, and ${ }^{2}$ Departments of Medicine, ${ }^{3}$ Pharmacology at Faculty of Medicine, \\ ${ }^{4}$ Psychobiology at Faculty of Psychology, University of Málaga, Málaga 29071, Spain, and ${ }^{5}$ Centro Investigación Biomédica en Red para Enfermedades \\ Neurodegenerativas, Institute of Health Carlos III, Madrid 28031, Spain
}

Working memory (WM) is a process of actively maintaining information in the mind for a relatively short period of time, and prefrontal cortex (PFC) has been thought to play a central role in its function. However, our understanding of underlying molecular events that translate into WM behavior remains elusive. To shed light on this issue, we have used three distinct nonhuman primate models of WM where each model represents three WM conditions: normal control, WM-deficient, and recuperated to normal from WM deficiency. Based on the hypothesis that there is a common molecular substrate for the coding of WM behavior, we have studied the relationship of these animals' performance on a WM task with their PFC levels of molecular components associated with Gq-phospholipase C and cAMP pathways, with the idea of identifying the footprints of such biomolecules. We observed that in all of the primate models WM deficiency was strongly related to the reduced concentration of $\mathrm{IP}_{3}$ in $\mathrm{PFC}$, whereas recuperation of WM-deficient animals to normal condition was associated with the normalization in $\mathrm{IP}_{3}$ level. However, this correlation was absent or weak for cAMP, active protein kinase A, dopamine $\mathrm{D}_{1}$ receptor, and Gq protein. In addition, WM deficiency related not only to pharmacological conditions but also to aging. Thus, it is suggested that optimal IP ${ }_{3}$ activity is essential for normal WM function and the maintenance of intracellular $\mathrm{IP}_{3}$-mediated $\mathrm{Ca}^{2+}$ level in PFC may serve as biochemical substrate for the expression of WM behavior.

\section{Introduction}

Prefrontal cortex (PFC) has been thought to be instrumental in working memory (WM) processing (Baddeley, 1992), and dysfunction in this area leads to an impairment in WM function (Fuster, 1997). Damage in PFC of nonhuman primate produces profound loss in the performance of animals on WM tasks (Butters et al., 1971; Passingham, 1985). Furthermore, demonstration of almost identical cue, delay, and response-related properties in neuronal populations of PFC, together with the observation of their sustained firing during the delay period, suggest that the expression of WM behavior primarily depends on activities in this area of brain (Funahashi et al., 1989; Chafee and GoldmanRakic, 1998). Consistent with this, imaging studies have also shown higher activity in PFC during performance on WM tasks (Wagner et al., 2001). An enhanced stimulus-specific spiking activity during the delay period has been considered to be a neuronal correlate of WM, and signaling via the Gq protein-phospholipase C (PLC) pathway is thought to be important for this activity (Runyan et al., 2005; Dash et al., 2007). However, the neurochemical substrate that serves the expression of WM behavior remains an enigma. Here, using three models with marked deficit

Received Sept. 15, 2009; revised Nov. 19, 2009; accepted Jan. 12, 2010.

This work was supported by Ministerio de Ciencia e Innovacion Grant BFU 06-0306 and Junta de Andalucia Grant CTS 586/08 to Z.U.K.

Correspondence should be addressed to Dr. Zafar U. Khan, Laboratoria Neurobiología, CIMES, Facultad de Medicina, Universidad de Málaga, Málaga 29071, Spain. E-mail: zkhan@uma.es.

DOI:10.1523/JNEUROSCI.4565-09.2010

Copyright $\odot 2010$ the authors $\quad$ 0270-6474/10/303067-05\$15.00/0 in WM, two models after recuperation from WM loss, and vehicle-treated matching control animals (see Table 1), we have studied the correlation of animal performance on WM task with PFC IP 3 , an end product of the Gq-PLC pathway, together with other biological components of Gq-PLC and cAMP pathways. The advantage of using several WM models was that it permitted us to map the footprints of biomolecules associated with WM in a stepwise manner from one WM condition to another within a model and across all models. Biomolecules showing similar levels in control normal and recovered normal animals but change in the WM-deficient condition of all models were considered highly correlated to WM. Our results show a strong correlation of the WM status of nonhuman primates with $\mathrm{IP}_{3}$ level in PFC not only in pharmacological conditions but also in aging.

\section{Materials and Methods}

Subjects. Adult Rhesus monkeys 5-7 years of age or old monkeys 18-22 years of age were used in this study. Animals were maintained in accordance with the guidelines of local Animal Experimentation, Welfare and Protection Committee and European Union Law (Council directive 86/ 609/EEC adopted in 1986 and the Protocol to the Amsterdam Treaty).

All monkeys were trained on a spatial working memory task in the Wisconsin General Testing Apparatus (WGTA) according to methods described previously (Goldman et al., 1970). Animals were always tested at the same time of day immediately before feeding. Highly palatable food rewards (e.g., a piece of peanut or miniature chocolate chips) were used during testing to minimize the need for dietary regulation. Animals performing $75-85 \%$ correctly (in the case of young animals) and 65- 
Table 1. Monkeys' WM condition on behavioral test before killing

\begin{tabular}{ll}
\hline Animal groups and their treatments & WM status $^{a}$ \\
\hline Amphetamine young animal group & \\
Vehicle control & Normal \\
Amphetamine treatment & Deficit \\
Amphetamine-treated animals 6 mo after withdrawal & Recuperated to normal \\
Haloperidol young animal group & \\
Vehicle control & Normal \\
Haloperidol treatment & Deficit \\
Haloperdol-treated animals after $\mathrm{D}_{1}$ agonist administration & Recuperated to normal \\
Haloperidol aged animal group & \\
Vehicle control & Normal \\
Haloperidol treatment & Deficit
\end{tabular}

${ }^{a}$ The WM status was determined by the results of animal performance on spatial delayed response shown in Figure 1A. Animals with significant reduction in the percentage of correct choices were considered to be WM deficient.

$70 \%$ correctly (in the case of old animals) with a $5 \mathrm{~s}$ delay period were used for the treatments and further studies.

WM behavioral test. Performance on a spatial delayed response task was used to assess the WM status of all the animals throughout the study. To execute the spatial delayed response task, one of the two food wells in the WGTA was baited in view of the monkey, then both wells were covered with identical plaques and an opaque screen was lowered for $5 \mathrm{~s}$. During the delay period, the monkey had to hold in mind the spatial location of the baited well to respond appropriately and be rewarded. After the delay, the animal had to move the correct plaque to obtain the reward. Animals were required to perform 30 trials per test session. Initial values were obtained from a mean of three consecutive test sessions corresponding to each monkey, and then these values were used for the calculation of the percentage of correct choice, as shown in Figure $1 \mathrm{~A}$ and Table 1 for each group.

WM models. Using a recipe from published articles, three WM models of both young and old nonhuman primates were prepared. A summary of the treatment of animals and their corresponding WM status is described in Table 1. Animals that showed significant reduction in performance on spatial delayed response task after treatment with either haloperidol or amphetamine were considered WM deficient (Table 1). All of the animals included in this study responded to the drug treatment.

Amphetamine-based WM models. Ten adult monkeys received intermittent, escalating doses $(0.1-1.0 \mathrm{mg} / \mathrm{kg}$ body weight) of amphetamine (from Sigma-RBI) via intramuscular injections for 6 weeks (Castner et al., 2005; Selemon et al., 2007). Forty-five days after withdrawal, when a significant loss in WM was observed (see Fig. $1 A$ ), a group of five animals was killed. Another group of five animals was killed 6 months after withdrawal, at which time animal performance on WM tasks returned to normal (see Fig. $1 \mathrm{~A}$ ).

Haloperidol-based WM models. A powder form of haloperidol (Sigma$\mathrm{RBI}$ ) in fruit was administered daily to nine adult monkeys at a dose of $0.07 \mathrm{mg} / \mathrm{kg}$ body weight during the first month, which was increased to $0.14 \mathrm{mg} / \mathrm{kg}$ body weight in the second month (Castner et al., 2000). Haloperidol-mediated WM deficits emerged between 3 and 4 months. A group of five animals was killed 4 months after the initiation of drug treatment when a substantial WM loss was observed (see Fig. $1 \mathrm{~A}$ ). The other group of four monkeys was continued with the treatment and was coadministered a selective $\mathrm{D}_{1}$ agonist ABT 431 (Lilly) at a dose of 0.0001 $\mathrm{mg} / \mathrm{kg}$ body weight for five consecutive days. This $\mathrm{D}_{1}$ agonist treatment has been shown to produce a progressive and long-lasting reversal of haloperidol-induced WM deficits (Castner et al., 2000). Once the animals showed normal WM status (see Fig. $1 A$ ), they were killed.

In another set of four aged monkeys, haloperidol was started by administration of daily doses of $0.20 \mathrm{mg} / \mathrm{kg}$ body weight and was increased to 0.27 and $0.35 \mathrm{mg} / \mathrm{kg}$ body weight at 2 week intervals. The latter dose was maintained until the time of killing. Once the WM deficit was observed (see Fig. $1 A$ ), animals were killed.

Brain tissues. After 2-6h of determination of behavioral status (Table 1; see Fig. $1 A$ ), animal brains were removed and small blocks of the dorsolateral prefrontal cortex (area 46) and striatum (caudate-putamen) were frozen in liquid $\mathrm{N}_{2}$ and stored at $-80^{\circ} \mathrm{C}$.
Homogenate and membrane preparation. Collected brain tissues were homogenized in Tris- $\mathrm{HCl}, \mathrm{pH} 7.4$, containing a mixture of protease inhibitors (Sigma-Aldrich) and were processed for the membrane preparation, as described previously (Khan et al., 1994, 1998; Khan and Gutierrez, 2004). In brief, homogenates were centrifuged at $3000 \mathrm{rpm}$ $(1075 \times g)$ for $10 \mathrm{~min}$ in an RC5C centrifuge (Sorvall). The resultant supernatant was centrifuged at 48,100 rpm $(105,000 \times g)$ for $1 \mathrm{~h}$ in an XL-90 Ultracentrifuge from Beckman to collect the membrane fraction as a pellet. Membrane fractions were washed twice in $50 \mathrm{~mm}$ Tris- $\mathrm{HCl}$, $\mathrm{pH} 7.4$, and were finally suspended in the same buffer and aliquoted. Aliquots were stored at $-80^{\circ} \mathrm{C}$ until used. The protein concentration was determined by the Lowry method.

$I P_{3}$ assay. A $0.5 \mathrm{ml}$ aliquot of homogenate from above in membrane preparation was mixed with the same amount of cold 15\% TCA. After 10 min on ice, it was centrifuged at $5000 \mathrm{rpm}$ for $15 \mathrm{~min}$ in a Microfuge. The collected supernatant was extracted three times with 10 volumes of diethyl ether saturated with water. The supernatant was then neutralized to $\mathrm{pH} 7.5$ by titration with $1 \mathrm{M} \mathrm{NaHCO} 3 . \mathrm{IP}_{3}$ was separated by a solid-phase procedure using Amprep SAX minicolumns (GE Healthcare). Briefly, columns were washed with $1 \mathrm{M} \mathrm{KHCO} 3$ followed by $15 \mathrm{ml}$ of water. After the samples were applied to the column, the column was washed with water and then with $0.1 \mathrm{ml}$ of $\mathrm{KHCO}$. The $\mathrm{IP}_{3}$ fraction was eluted with $5 \mathrm{ml}$ of $0.17 \mathrm{M} \mathrm{KHCO}$. The determination of $\mathrm{IP}_{3}$ concentration in collected fractions was performed by D-myoinositol 1,4,5-triphosphate $\left[{ }^{3} \mathrm{H}\right]$ assay system from GE Healthcare.

cAMP immunoassay. In $0.5 \mathrm{ml}$ of prepared homogenate from homogenate and membrane preparation, $0.5 \mathrm{ml}$ of Hank's buffer was added, followed by the addition of $1.0 \mathrm{ml}$ of acetonitrile. The samples were mixed, incubated on ice for $10 \mathrm{~min}$, and centrifuged at $6000 \mathrm{rpm}$ for $10 \mathrm{~min}$. The collected supernatants were loaded on conditioned Amprep SAX minicolumns. Samples were eluted by $3.0 \mathrm{ml}$ of methanol with $0.1 \mathrm{M} \mathrm{HCl}$ and dried under nitrogen. The cAMP assay was performed as described by the manufacturer using the cAMP enzyme immunoassay system from GE Healthcare.

Immunoblots. Homogenates (for active PKA levels) and prepared membrane fractions (for $\mathrm{G} \alpha \mathrm{q}$ protein levels) were treated with SDS buffer and processed for Western blot analysis similar to as described previously (Khan et al., 2000, 2001; López-Aranda et al., 2006). Homogenate proteins (395 $\mu \mathrm{g} / \mathrm{lane})$ and membrane proteins (100 $\mu \mathrm{g} / \mathrm{lane})$ were separated by $12 \%$ SDS-PAGE and transferred onto a polyvinylidene fluoride membrane (GE Healthcare). The membranes were blocked with $5 \%$ milk in $10 \mathrm{~mm}$ Tris- $\mathrm{HCl}, \mathrm{pH} 7.5$ containing $0.05 \%$ Tween-20 and $0.2 .9 \% \mathrm{NaCl}$ (TTBS) for $15 \mathrm{~min}$ and washed with TTBS, followed by incubation with either mouse monoclonal antibody to active PKA (1: 1000 dilution in TTBS with 2\% BSA; Transduction Laboratories) or affinity-purified rabbit polyclonal antibodies to $\mathrm{G} \alpha \mathrm{q}$ (1:500 dilution in TTBS with 2\% BSA; Santa Cruz Biotechnology). Corresponding rabbit and mouse secondary antibodies coupled to HRP (GE Healthcare) were diluted to 1:2500 in TTBS with 2\% BSA and 5\% nonfat dry milk, and incubated for $1 \mathrm{~h}$. Development of the immunoreactivity was performed with ECL Western Blotting Detection Reagents (GE Healthcare) according to the manufacturer protocol. For the analysis of immunoreactive bands, we used ScanMaker 9800XL (Microtek) to scan the film and program Visilog for the determination of optical density. Relative gray values were obtained from the scale of $0-255$ preset in the program. Vehicle control of amphetamine group was considered 100\%.

Receptor-ligand binding assay. Membrane-bound dopamine $\mathrm{D}_{1}$ receptors were assayed by $\left[{ }^{3} \mathrm{H}\right] \mathrm{SCH} 23390$ binding, as described previously (Khan et al., 1998, 2000). Briefly, $200 \mu \mathrm{g}$ of membrane protein was incubated with $0-20 \mathrm{~nm}$ radioligand for $1 \mathrm{~h}$ at $22^{\circ} \mathrm{C}$ in a total volume of $0.5 \mathrm{ml}$ of $50 \mathrm{~mm}$ Tris- $\mathrm{HCl}$ (pH 7.4) containing 1 mм EDTA, $5 \mathrm{~mm} \mathrm{KCl,} 1.5 \mathrm{~mm}$ $\mathrm{CaCl} 2,4 \mathrm{~mm} \mathrm{MgCl}$, and $120 \mathrm{~mm} \mathrm{NaCl}$. The reaction was stopped by rapid filtration through $0.3 \%$ polyethylenimine-soaked Whatman GF/B filters and washed three times with $50 \mathrm{~mm}$ Tris- $\mathrm{HCl}, \mathrm{pH}$ 7.4. Filters were dried and counted for retained radioactivity. Nonspecific binding was determined by performing the assay in the presence of $1000 \times$ of either $(+)$ butaclamol- $\mathrm{HCl}$ or fluphenazine. Background activity was subtracted from total activity, which was $5-7 \%$.

Data analysis. Digital images of immunoblots were analyzed by densitometry using Visilog 6. Values are presented as the mean \pm SEM of four 

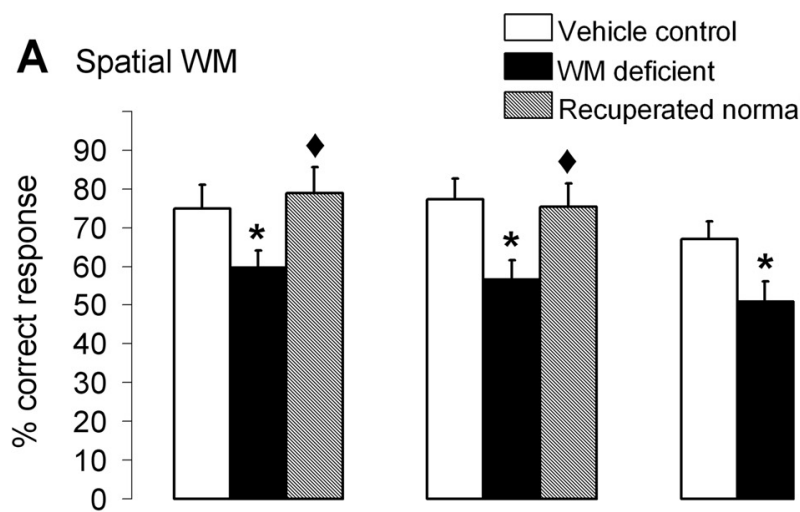

\section{B IP3}
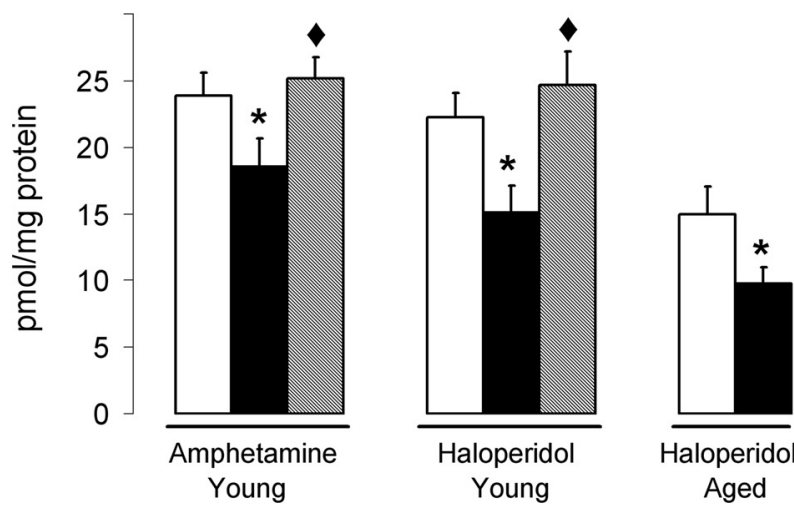

Figure 1. WM status of nonhuman primate models before they were killed and their $\mathrm{PFCIP}$ concentration. $\boldsymbol{A}$, As shown previously (Castner et al., 2000, 2005), both amphetamine (black bars in amphetamine young group) and haloperidol (black bars in haloperidol young and haloperidol aged groups) treatments produced significant loss in WM. Six months after withdrawal in amphetamine-treated monkeys (Castner et al., 2005) (hatched bars in amphetamine young group) and after dopamine $\mathrm{D}_{1}$ agonist administration to haloperidol-treated monkeys (Castner et al., 2000) (hatched bars in haloperidol young group) the WM deficit observed in animals recovered to a normal level. $\boldsymbol{B}_{1}$ A significant reduction in the amount of $\mathrm{IP}_{3}$ was observed in animals with WM deficiency. Recuperation in WM was associated with the normalization in $\mathbb{P}_{3}$ concentration. * results are significantly different from the control group; ${ }^{\star}$ results are significantly different from the WM-deficient group.

to five animals. Statistical analysis of the physiologic variables was performed using Student's $t$ test. A probability value $<0.05$ was considered statistically significant.

\section{Results}

Three models with marked deficit in WM, two models after recuperation from WM loss and vehicle-treated matching control animals were included in this study (Table 1). Similar to previous reports, the treatment of young animals with amphetamine produced a significant loss in WM; however, 6 months after withdrawal these animals returned to normal condition (Castner et al., 2005) (Fig. 1A). In another experiment, exposure of both young and aged animals with haloperidol also resulted in a marked reduction in WM (Castner et al., 2000) (Fig. 1 A). Further administration of $\mathrm{D}_{1}$ agonist $\mathrm{ABT} 431$ to haloperidol-induced, WM-deficient young animals resulted in the reversal of WM loss observed in these animals (Castner et al., 2000) (Fig. 1A). After confirming WM status (Table 1), all the animals were killed and their PFCs were processed for neurochemical study. Animals with WM deficiency developed after treatment with either amphetamine in young animals or with haloperidol in young and aged animals had significantly lower $\mathrm{IP}_{3}$ levels (Fig. $1 \mathrm{~B}$ ). A lower

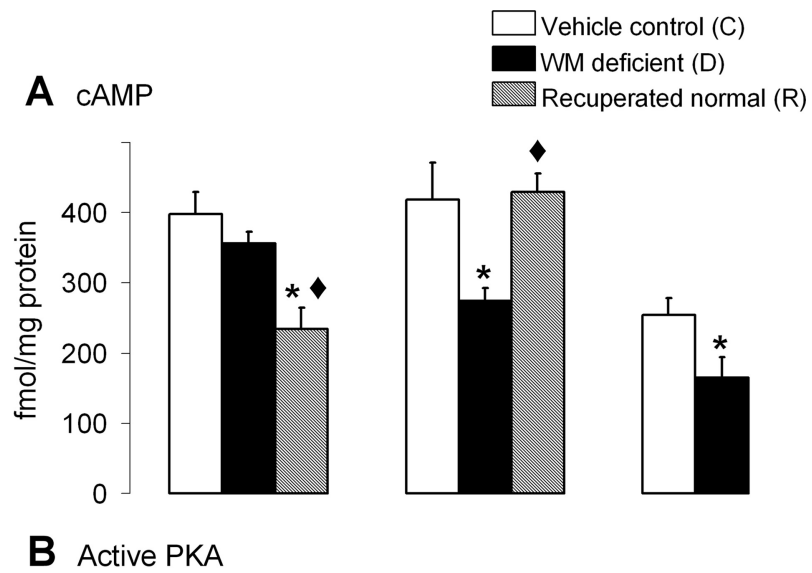

[i] Blots

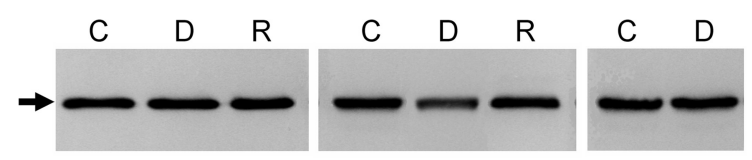

[ii] Optical density plot

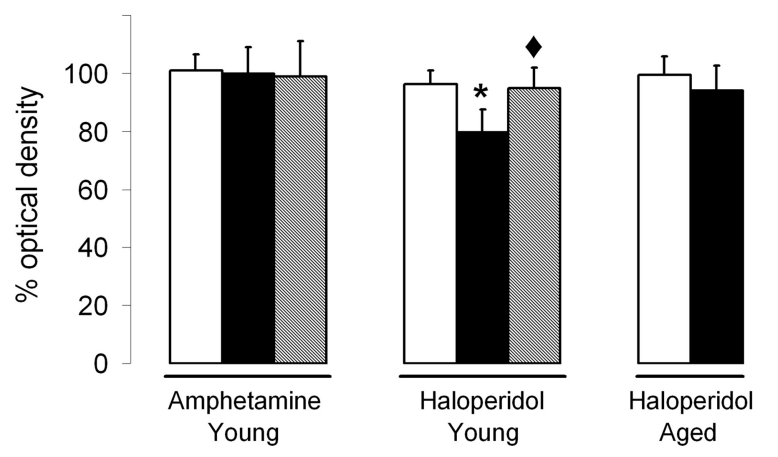

Figure 2. Levels of CAMP and active PKA in PFC. $\boldsymbol{A}$, The CAMP level in amphetamine young group did not coincide with the behavioral performance seen in these animals; however, there were coincidence in cases of haloperidol young and haloperidol aged animal groups where WM-deficient animals showed reduced cAMP levels. Bi, ii, Representative blots of active PKA (i) and their optical density plot (ii) are shown. The amphetamine young and haloperidol aged animal groups did not show any change in active PKA level. However, a reduction in WMdeficient animals and an increase in recuperated normal animals of the haloperidol young group were observed. The arrow in the blots in $i$ indicates the immunoreactive band of $39 \mathrm{kDa}$ corresponding to a catalytically active subunit of PKA protein size. * results are significantly different from control group; ${ }^{\star}$ results are significantly different from WM-deficient animals.

$\mathrm{IP}_{3}$ level was also observed in normal aged animals compared with normal young animals (Fig. $1 B$ ). Most importantly, we found that the recovery in WM after either $D_{1}$ agonist administration to haloperidol-treated monkeys or 6 months after withdrawal to amphetamine-treated young animals was associated with an increase in $\mathrm{IP}_{3}$ level (Fig. $1 B$ ). In all the $\mathrm{WM}$ models, there was strong correlation between the level of animal performance on the WM task and the $\mathrm{IP}_{3}$ concentration in PFC. However, this strong correlation was absent or weak for other components associated with cAMP (Fig. $2 A, B$ ) and Gq-PLC pathways (Fig. $3 A, B)$. In contrast to $\mathrm{PFC}, \mathrm{IP}_{3}$ level in the striatum showed no correlation with WM (Fig. 4). cAMP and active PKA, main components of the cAMP pathway, showed no relation with WM in amphetamine young and haloperidol aged animals (Fig. 2A,B). However, a correlation was observed in the haloperidol young group of animals. An inverse relation of WM with both cAMP 


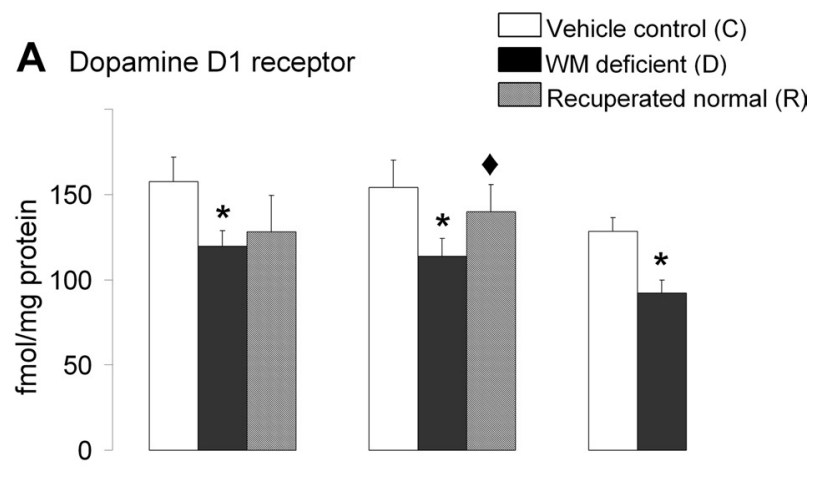

B Gq protein

[i] Blots

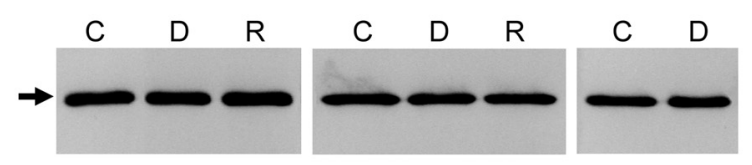

[ii] Optical density plot

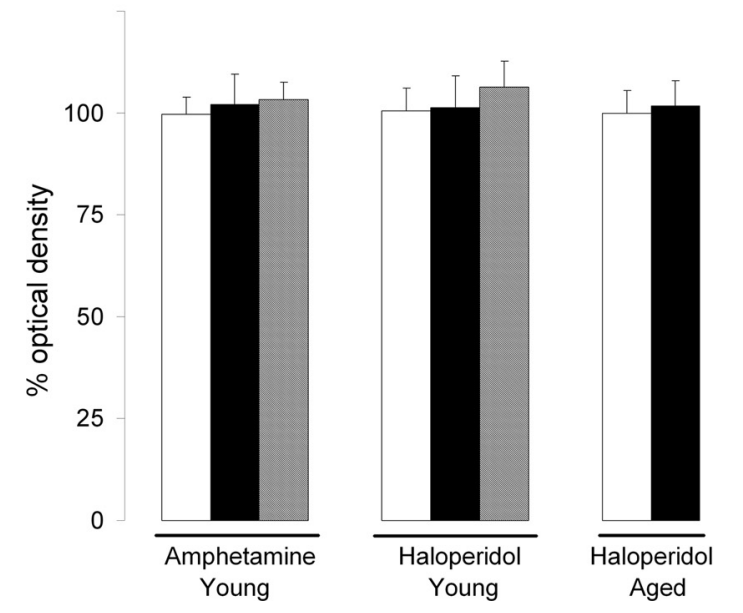

Figure 3. Amount of dopamine $D_{1}$ receptor and $G q$ protein in PFC. $A, A$ correlation of $D_{1}$ receptor level with WM status was seen in WM-deficient animals of all the groups but this correlation was lacking in the recuperated normal animals of amphetamine young group. $\mathbf{B i}$, ii, Representative blots of $\mathrm{Gq}$ protein (i) and their optical density plot (ii) are shown. $\mathrm{Gq}$ protein level was not changed in any of the groups. The arrow in the blots in $i$ indicates the immunoreactive polypeptide band of $42 \mathrm{kDA}$ corresponding to $\mathrm{Gq}$ protein size. * results are significantly different from control group; results are significantly different from WMdeficient animals.

(Dash et al., 2007; Wang et al., 2007) and higher PKA activity (Taylor et al., 1999) has been shown previously. Thus, the changes observed in components related to the cAMP pathway of the haloperidol group of animals do not actually reflect an association with WM. In contrast to the cAMP pathway, activation of dopamine receptor is known to enhance the WM in nonhuman primates (Castner et al., 2000; Castner and Goldman-Rakic, 2004). Our results show that the level of dopamine $D_{1}$ receptor in WM-deficient animals in both the amphetamine young and haloperidol young and old groups were significantly reduced (Fig. $3 A$ ). The recovery in WM of haloperidol young animals was seen with the increase in dopamine $\mathrm{D}_{1}$ receptor concentration. However, this correlation was lacking in amphetamine young animals, where no change was observed (Fig. $3 A$ ). In contrast to the dopamine $D_{1}$ receptor, $G q$ protein, another component of the Gq-PLC pathway, was unchanged in all the animal groups (Fig. 3B).

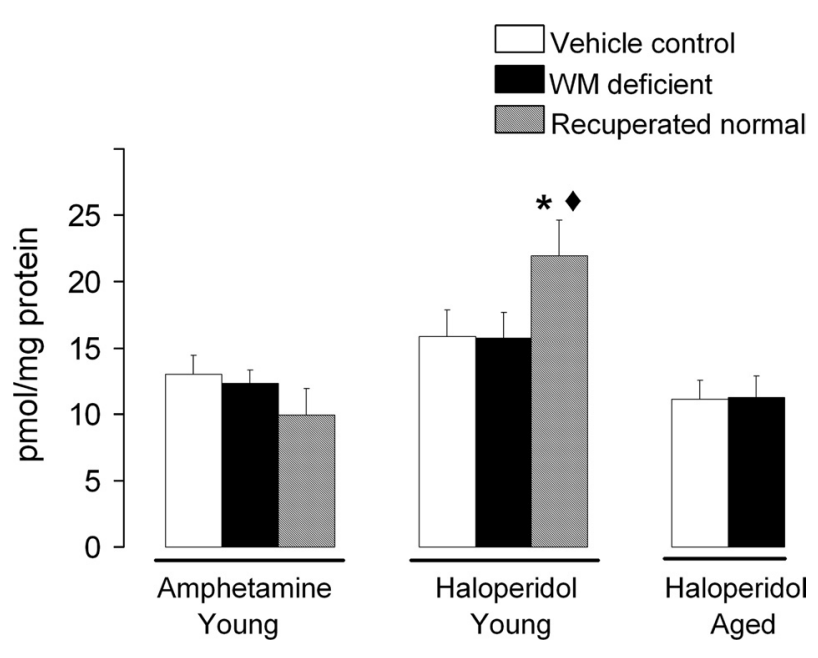

Figure 4. $\quad \mathbb{P}_{3}$ concentration in the striatum. $\mathbb{P}_{3}$ level in this area did not correlate with the WM status seen in the animals. An increase in $\mathbb{P}_{3}$ was observed in recuperated normal animals of the haloperidol young group. However, this may be due to the activation of dopamine $D_{1}$ receptor while $D_{1}$ agonist is on board in these animals. Values are expressed as the mean \pm SEM of four to five animals. * results are significantly different from control; $\bullet$ results are significantly different from WM-deficient animals.

\section{Discussion}

The activation of cAMP-PKA pathway has an inverse relation with WM (Dash et al., 2007; Wang et al., 2007). An increase in cAMP concentration or activation of PKA is expected to reduce the level of animal performance on a WM task. Therefore, the absence of correlation of cAMP and active PKA with WM suggests that the CAMP-PKA pathway may not be responsible for the expression of WM behaviors seen in nonhuman primates. However, on the other hand, the results of the activation of Gq protein pathway during performance on WM task and the finding that the inhibition of PLC impairs WM (Runyan et al., 2005) suggest a directly proportional relation of Gq-PLC pathway with WM. Therefore, lower WM performance in WM-deficient animals was perhaps due to lower activity in this pathway. Activation of Gq protein-coupled receptors, such as dopamine $\mathrm{D}_{1}$ receptor, which are known to influence WM functions (Dash et al., 2007), leads to the production of $\mathrm{IP}_{3}$ and diacylglycerol through the Gq-PLC pathway. The $\mathrm{IP}_{3}$, then, releases $\mathrm{Ca}^{2+}$ from intracellular stores. It has been shown that stimulation of Gq-coupled dopamine $D_{1}$ receptor depresses recurrent excitatory transmission and generates a substantial and prolonged attenuation of $\mathrm{IP}_{3}$ signaling (Abdul-Ghani et al., 1996), a process shown to be necessary for the mediation of WM functions (Goldman-Rakic, 1995). Therefore, $\mathrm{IP}_{3}$ deficit in $\mathrm{WM}$-deficient monkeys may reflect a chronic state of depressed neuronal transmission. A requirement of $\mathrm{Ca}^{2+}$ release from intracellular stores via $\mathrm{IP}_{3}$ for a persistent recurrent excitation in prefrontal neurons (Gao and Goldman-Rakic, 2006) further indicates the importance of optimal $\mathrm{IP}_{3}$ signaling for normal WM function. In addition, schizophrenics, patients with dominant WM deficit, exhibit low levels of PFC RGS 4, an inhibitor of Gq protein-induced intracellular $\mathrm{Ca}^{2+}$ release (Mirnics et al., 2001). It is proposed that $\mathrm{IP}_{3}$-mediated intracellular $\mathrm{Ca}^{2+}$ in PFC may provide a biochemical environment that is crucial for the maintenance of information during the delay period, a period when the animal has to hold the information on stimulus for few seconds in the mind before responding. In summary, our results show a strong link between the $\mathrm{IP}_{3}$ concentrations in PFC and WM performance in nonhuman primates. 


\section{References}

Abdul-Ghani MA, Valiante TA, Carlen PL, Pennefather PS (1996) Metabotropic glutamate receptors coupled to IP3 production mediate inhibition of IAHP in rat dentate granule neurons. J Neurophysiol 76:2691-2700.

Baddeley A (1992) Working memory. Science 255:556-559.

Butters N, Pandya D, Sanders K, Dye P (1971) Behavioral deficits in monkeys after selective lesions within the middle third of sulcus principalis. J Comp Physiol Psychol 76:8-14.

Castner SA, Goldman-Rakic PS (2004) Enhancement of working memory in aged monkeys by a sensitizing regimen of dopamine $D_{1}$ receptor stimulation. J Neurosci 24:1446-1450.

Castner SA, Williams GV, Goldman-Rakic PS (2000) Reversal of antipsychotic-induced working memory deficits by short-term dopamine D1 receptor stimulation. Science 287:2020-2022.

Castner SA, Vosler PS, Goldman-Rakic PS (2005) Amphetamine sensitization impairs cognition and reduces dopamine turnover in primate prefrontal cortex. Biol Psychiatry 57:743-751.

Chafee MV, Goldman-Rakic PS (1998) Matching patterns of activity in primate prefrontal area $8 \mathrm{a}$ and parietal area 7ip neurons during a spatial working memory task. J Neurophysiol 79:2919-2940.

Dash PK, Moore AN, Kobori N, Runyan JD (2007) Molecular activity underlying working memory. Learn Mem 14:554-563.

Funahashi S, Bruce CJ, Goldman-Rakic PS (1989) Mnemonic coding of visual space in the monkey's dorsolateral prefrontal cortex. J Neurophysiol 61:331-349.

Fuster JM (1997) Network memory. Trends Neurosci 20:451-459.

Gao WJ, Goldman-Rakic PS (2006) NMDA receptor-mediated epileptiform persistent activity requires calcium release from intracellular stores in prefrontal neurons. Exp Neurol 197:495-504.

Goldman PS, Rosvold HE, Mishkin M (1970) Evidence for behavioral impairment following prefrontal lobectomy in the infant monkey. J Comp Physiol Psychol 70:454-463.

Goldman-Rakic PS (1995) Cellular basis of working memory. Neuron 14:477-485.

Khan ZU, Gutierrez A (2004) Distribution of C-terminal splice variant of G alpha i2 in rat and monkey brain. Neuroscience 127:833-843.

Khan ZU, Gutiérrez A, De Blas AL (1994) Short and long form gamma 2 subunits of the GABAA/benzodiazepine receptors. J Neurochem 63:14661476

Khan ZU, Mrzljak L, Gutierrez A, de la Calle A, Goldman-Rakic PS (1998) Prominence of the dopamine D2 short isoform in dopaminergic pathways. Proc Natl Acad Sci U S A 95:7731-7736.

Khan ZU, Gutiérrez A, Martín R, Peñafiel A, Rivera A, de la Calle A (2000) Dopamine D5 receptors of rat and human brain. Neuroscience 100:689699.

Khan ZU, Koulen P, Rubinstein M, Grandy DK, Goldman-Rakic PS (2001) An astroglia-linked dopamine D2-receptor action in prefrontal cortex. Proc Natl Acad Sci U S A 98:1964-1969.

López-Aranda MF, Acevedo MJ, Carballo FJ, Gutiérrez A, Khan ZU (2006) Localization of the GoLoco motif carrier regulator of G-protein signalling 12 and 14 proteins in monkey and rat brain. Eur J Neurosci 23:2971-2982.

Mirnics K, Middleton FA, Stanwood GD, Lewis DA, Levitt P (2001) Disease-specific changes in regulator of G-protein signaling 4 (RGS4) expression in schizophrenia. Mol Psychiatry 6:293-301.

Passingham RE (1985) Memory of monkeys (Macaca mulatta) with lesions in prefrontal cortex. Behav Neurosci 99:3-21.

Runyan JD, Moore AN, Dash PK (2005) A role for prefrontal calciumsensitive protein phosphatase and kinase activities in working memory. Learn Mem 12:103-110.

Selemon LD, Begović A, Goldman-Rakic PS, Castner SA (2007) Amphetamine sensitization alters dendritic morphology in prefrontal cortical pyramidal neurons in the non-human primate. Neuropsychopharmacology 32:919-931.

Taylor JR, Birnbaum S, Ubriani R, Arnsten AF (1999) Activation of cAMPdependent protein kinase A in prefrontal cortex impairs working memory performance. J Neurosci 19:RC23.

Wagner AD, Maril A, Bjork RA, Schacter DL (2001) Prefrontal contributions to executive control: fMRI evidence for functional distinctions within lateral prefrontal cortex. Neuroimage 14:1337-1347.

Wang M, Ramos BP, Paspalas CD, Shu Y, Simen A, Duque A, Vijayraghavan S, Brennan A, Dudley A, Nou E, Mazer JA, McCormick DA, Arnsten AF (2007) Alpha2A-adrenoceptors strengthen working memory networks by inhibiting cAMP-HCN channel signaling in prefrontal cortex. Cell 129:397-410. 